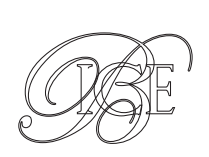

\title{
ESTRATEGIA NACIONAL INDUSTRIA CONECTADA 4.0 Iniciativas y programas
}

Conscientes de la importancia y trascendencia de la transformación digital para nuestra industria, la Secretaría General de Industria y de la Pyme está implementando desde 2015 la Estrategia Nacional Industria Conectada 4.0.

La estrategia nacional surge de un proceso participativo en el que los sectores público y privado no solo analizan los retos de la industria ante los desafíos de la cuarta revolución industrial, sino que ofrecen un conjunto de acciones y programas específicos para facilitar la transformación de las empresas industriales hacia su total digitalización.

Este artículo detalla pormenorizadamente los elementos constitutivos de la Estrategia Nacional Industria Conectada 4.0 y su estado de situación.

Palabras clave: transformación digital, cuarta revolución industrial, política industrial.

Clasificación JEL: L52, O38.

\section{Introducción}

La denominada Industria 4.0 surge como una nueva revolución industrial que incorpora nuevas tecnologías o habilitadores digitales a la industria. Una transformación digital que ya está afectando a todas las empresas, pues, más pronto o más tarde, todas ellas se verán avocadas a adaptarse y reorientarse hacia un modelo industrial en el que la innovación es colaborativa, los medios productivos están conectados, las cadenas de suministro están integradas y los canales de distribución y

*Este artículo ha sido elaborado por Fernando Carabias Herrera, Jefe de Servicio; por Montserrat Carrasco Morales, Jefe de Servicio; y por Ana García González. Jefe de Área.

Versión de mayo de 2020.

DOI: https:/doi.org/10.32796/bice.2020.3126.7070 atención son digitales. Por todo ello, la transformación digital supone todo un desafío para la industria, al tiempo que ofrece una gran oportunidad para mejorar la competitividad empresarial.

En el año 2015 se puso en marcha, desde la Secretaría General de Industria y de la Pyme (SGIPYME), la Estrategia Industria Conectada 4.0 con el fin de impulsar la transformación digital de la industria.

La estrategia nacional, alineada con las directrices europeas e integradora de las estrategias desplegadas por las comunidades autónomas (CC AA), promueve tres objetivos claros y ambiciosos, a saber:

1. Incrementar el valor añadido industrial y el empleo cualificado en el sector. 


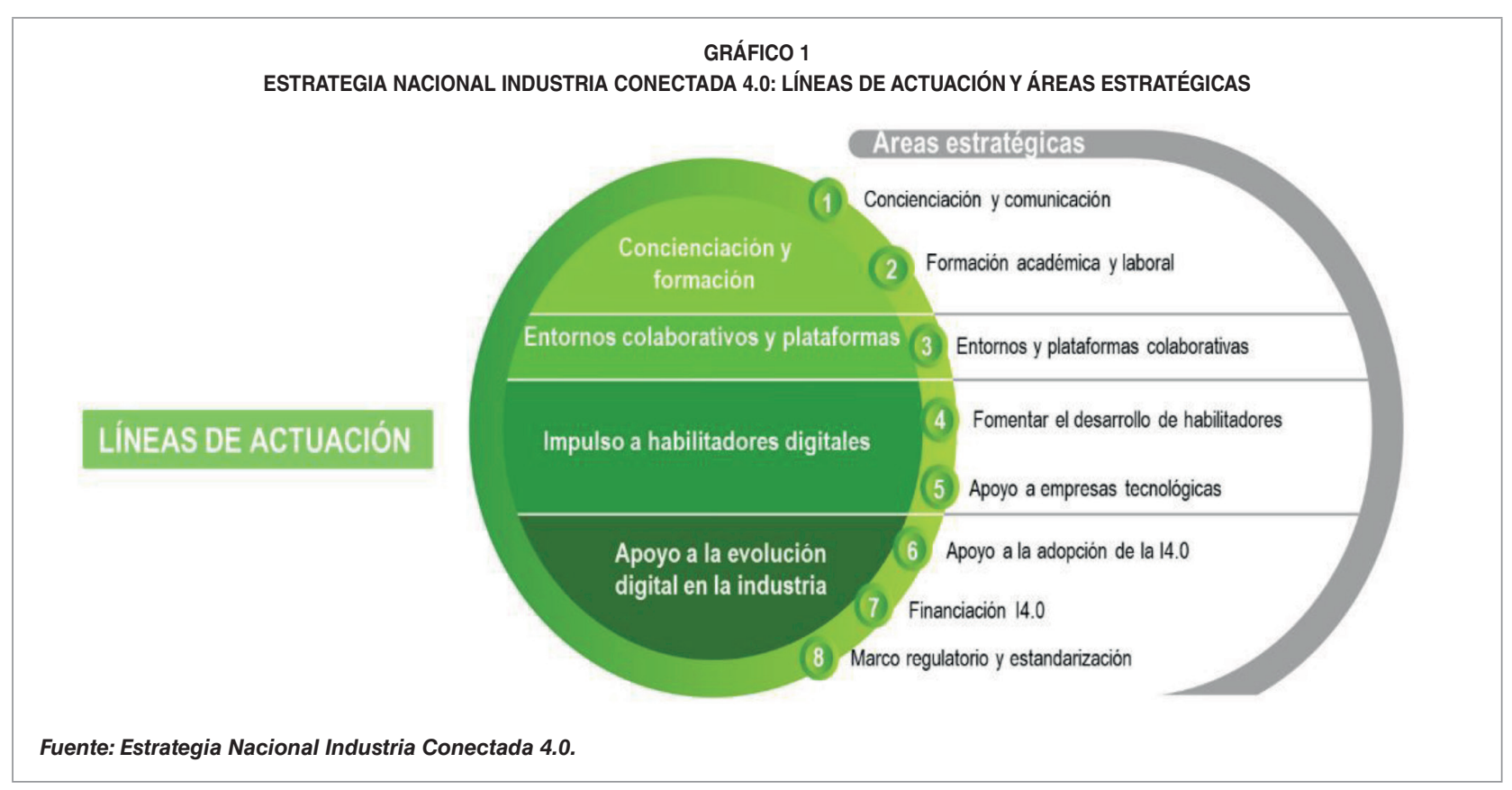

2. Favorecer un modelo industrial de futuro para la industria española, con el fin de potenciar los sectores venideros y aumentar su potencial de crecimiento.

3. Desarrollar palancas competitivas diferenciales para favorecer la industria española e impulsar sus exportaciones.

Esta estrategia presenta como rasgos más distintivos, por un lado, tener un carácter integral u holístico, es decir, tratar de dar respuesta al conjunto de áreas implicadas en el proceso de transformación digital y, por otro lado, posicionar a la empresa en el núcleo de su concepción y desarrollo.

Para apoyar a la empresa industrial en el proceso de trasformación digital, la estrategia nacional define cuatro líneas de actuación sobre las que se articulan ocho áreas estratégicas, concebidas como los pilares que facilitan la transición de nuestra industria hacia el paradigma de la cuarta revolución industrial.

El resto del artículo desarrolla los diferentes programas y actuaciones que se están impulsando desde la SGIPYME por área estratégica.

\section{2. Área de concienciación y comunicación}

El principal objetivo de las actuaciones de esta área es garantizar el conocimiento del concepto Industria 4.0 y de sus tecnologías asociadas, así como el desarrollo de competencias en Industria 4.0 en España.

\subsection{Página web Industria Conectada $4.0^{1}$}

Se ha creado una página web con contenidos adaptados a las necesidades de las empresas industriales: información sobre programas de apoyo, Congreso Industria Conectada 4.0, Premios Nacionales Industria Conectada 4.0, Catálogo de Iniciativas $\square$

1 www.industriaconectada40.gob.es 
Regionales para el fomento de la Industria 4.0 en España, eventos, estrategias e informes.

\subsection{Cuenta de Twitter}

La Estrategia de Industria Conectada 4.0 dispone de su propio perfil de Twitter (@IConectada40). Actualmente la cuenta dispone de más de cuatro mil seguidores.

\subsection{Boletín Informativo IC4.02}

Mensualmente se elabora un boletín informativo sobre Industria Conectada 4.0 que recoge los aspectos más relevantes de este ámbito: informes, artículos, noticias, convocatorias, etc. Se distribuye a más de 3.500 destinatarios de todos los sectores industriales.

\subsection{Catálogo de Iniciativas Regionales para el Fomento de la Industria 4.0 en España ${ }^{3}$}

Se trata de un documento de revisión periódica que aglutina los distintos instrumentos de fomento de la digitalización de la industria de la Administración General del Estado y las diferentes CC AA.

Este documento analiza y clasifica las iniciativas de las distintas comunidades en las siguientes áreas:

- Sensibilización, que comprende todas aquellas acciones dirigidas a la difusión,

2 https://www.industriaconectada40.gob.es/difusion/noticias/Paginas/ suscripcion. aspx?g=E5805D65-8485-4203-A5F2-AF9D47E5650E

3 https://www.industriaconectada40.gob.es/estrategias-informes/ Paginas/iniciativas-regionales.aspx formación y sensibilización del tejido empresarial, en cuanto a los beneficios, características e impactos que la Industria 4.0 puede llegar a tener en los diferentes sectores económicos.

- Estrategia, que analiza la existencia de un marco de referencia en el tiempo, bajo el cual se agrupan aquellas acciones orientadas a fomentar el impulso y la efectiva implantación de las soluciones y herramientas de la Industria 4.0 en cada región.

- Asesoramiento, dedicado a la puesta en marcha de servicios o programas de apoyo activo a la implantación de la Industria 4.0 en sectores y empresas de la región.

- Apoyo financiero, que analiza la existencia de un marco de apoyos financieros y ayudas a fondo perdido para el estímulo de acciones conducentes a la adopción de soluciones Industria 4.0.

Es la primera vez que se pone a disposición de las empresas industriales una herramienta de este tipo que aglutina todas las acciones de los diferentes niveles administrativos del Estado para apoyar a la industria.

\subsection{Congreso de Industria Conectada 4.0 $(\mathrm{CIC} 40)^{4}$}

El Ministerio de Industria, Comercio y Turismo, con la colaboración de la Agencia Española para la Calidad (AEC), organiza, desde 2017 y con periodicidad anual, el Congreso de Industria Conectada 4.0. Es uno de los instrumentos más relevantes del área estratégica de «Concienciación y comunicación».

4 https://www.industriaconectada40.gob.es/congreso/Paginas/ congreso-nacional.aspx 
El congreso ha conseguido aglutinar, en sus tres primeras ediciones, a los cuatro actores principales de la transformación digital: el sector industrial, el sector de los habilitadores digitales, el mundo de la consultoría y el conjunto de asociaciones empresariales.

Es un evento de referencia nacional, y con clara vocación internacional, sobre la transformación digital de la industria, que se ha convertido en un espacio de divulgación, sensibilización e intercambio de experiencias y retos sobre la Industria 4.0. Así lo corroboran los resultados obtenidos, como media, en las tres ediciones del congreso: alrededor de mil asistentes, más de quinientas inscripciones en streaming y más de treinta mil visitas a la web.

En la última edición, celebrada en 2019, se estrenó la figura del «país invitado» con el objetivo de fomentar la dimensión internacional del congreso y las relaciones económicas y comerciales entre los sectores industriales español y del «país invitado». La República de Corea fue el país invitado y estuvo representada al más alto nivel institucional (viceministro de Ciencia y TIC, director general del Departamento de Cooperación con Europa y África del Ministerio de Ciencia y TIC, presidente del Institute for Information and Communication Technology Promotion, embajador de la República de Corea en España y ministro consejero y de Asuntos Económicos de la embajada de la República de Corea en España).

\subsection{Premios Nacionales Industria Conectada $4.0^{5}$}

Con el fin de reconocer los esfuerzos y el mérito de las empresas industriales

\footnotetext{
5 https://www.industriaconectada40.gob.es/premios/Paginas/ premios.aspx
}

españolas en el ámbito de la digitalización, el Ministerio de Industria, Comercio y Turismo, a través de la SGIPYME, creó en el año 2019 los Premios Nacionales Industria Conectada $4.0^{6}$.

Los premios persiguen incrementar la concienciación del tejido industrial español sobre la necesaria transformación digital y fomentar la visibilidad de los instrumentos de apoyo en este proceso. A la vez, constituyen una herramienta de identificación, difusión y reconocimiento de proyectos españoles de éxito en la Industria 4.0, coadyuvando a incrementar las vocaciones industriales y digitales entre nuestros jóvenes.

Los premios consisten en un trofeo representativo y tienen carácter honorífico. Los premios correspondientes a la primera edición fueron entregados durante la celebración del III Congreso de Industria Conectada 4.0 por la ministra de Industria, Comercio y Turismo, María Reyes Maroto, en presencia del secretario general de Industria y de la Pequeña y Mediana Empresa, Raül Blanco Díaz, con el siguiente reparto:

- Premio Nacional Industria Conectada 4.0 a la Gran Empresa Industrial: IVECO ESPAÑA SL.

- Premio Nacional Industria Conectada 4.0 a la Pequeña y Mediana Empresa Industrial: FAMA SOFÁS SLU.

- Nominados en la modalidad de Premio Nacional Industria Conectada 4.0 a la Pequeña y Mediana Empresa Industrial: Bodegas Franco Españolas SA y Mecanizados y Montajes Aeronáuticos SA. $\triangleright$

6 Orden ICT/48/2019, de 21 de enero, por la que se crean los Premios Nacionales Industria Conectada 4.0 y se establecen las bases reguladoras para su organización, gestión y concesión (BOE n.ํ22, de 25.01.2019). 


\section{3. Área de formación académica y laboral}

El objetivo de esta área estratégica es desarrollar el capital humano con los conocimientos necesarios en el desarrollo, uso y aplicación de los habilitadores digitales, actuando tanto en el ámbito de la formación laboral como en el de la educación.

Las iniciativas y programas correspondientes a esta área se han puesto en marcha en colaboración con la Escuela de Organización Industrial (EOI). A título de ejemplo citamos algunos de los programas que se están desarrollado:

- Inmersión Digital en Industria 4.0. Programa formativo para directivos.

- Programas formativos de digitalización y asesoramiento pyme como parte de las medidas para contrarrestar los efectos COVID-19: Asesoramiento PYME, Asesoramiento express y PYME digital?.

- Programa de Inmersión Digital en Industria 4.0 COVID-198.

\section{4. Área de entornos y plataformas colaborativas}

Esta área se centra en fomentar la colaboración entre empresas de diversos sectores industriales, empresas tecnológicas, centros de investigación y otras entidades con el fin de promover el desarrollo de soluciones 4.0 adaptadas a las necesidades de la industria.

Las iniciativas y programas correspondientes a esta área son:

\footnotetext{
https://www.eoi.es/es/programasapoyocovid19

8 https://www.eoi.es/es/inmersiondigitalindustriacovid19
}

\subsection{Programa de apoyo a las Agrupaciones Empresariales Innovadoras $^{9}$}

El apoyo a las agrupaciones o clústeres innovadores es una de las fórmulas más adecuadas para impulsar y extender con rapidez la práctica de la innovación a empresas que difícilmente podrían acometer proyectos mínimamente relevantes si no es a través de fórmulas de colaboración. Las agrupaciones innovadoras pueden facilitar los procesos de innovación ligados a la digitalización de la industria entre las empresas que lo forman. El Ministerio de Industria apoya desde 2007 la actividad innovadora de estas agrupaciones a través del Programa de apoyo a las Agrupaciones Empresariales Innovadoras (AEI).

Las subvenciones totales concedidas entre 2007 y 2019 ascendieron a 75 millones de euros, correspondientes a 1.804 proyectos, con un presupuesto total de 132 millones de euros. La mayor parte de los recursos se concentraron en las comunidades autónomas de Cataluña, Aragón, Madrid, Andalucía, Comunidad Valenciana, Castilla y León, y Galicia; y en los sectores de TIC: agroalimentario, medioambiente y energía, maquinaria y tecnología industrial, automoción, hábitat, y biotecnología y salud.

Actualmente hay $83 \mathrm{AEI}$ inscritas en el Registro Especial de Agrupaciones Empresariales Innovadoras del ministerio, en las que participan unas 6.800 empresas, que emplean a 1,8 millones de trabajadores y colaboran con más de trescientos organismos de investigación y centros tecnológicos.

9 http://www.ipyme.org/es-ES/Financiacion/AEI/Paginas/AEINueva. aspx 


\subsection{Digital Innovation Hubs ${ }^{10}$}

En el marco del Programa Europa Digital, la Comisión Europea tiene previsto fomentar el desarrollo de una red europea de Digital Innovation Hubs (DIH). Los DIH tendrán un papel fundamental en el acceso y en la adopción, por parte de la industria, de las tecnologías digitales, en especial de la inteligencia artificial, la supercomputación y la ciberseguridad.

Un DIH es una organización o grupo de organizaciones coordinadas, sin ánimo de lucro, que ofrece conocimientos tecnológicos e instalaciones de ensayo y experimentación para permitir la transformación digital de la industria (fundamentalmente pymes) y del sector público, y que promueve la innovación abierta. El DIH estará compuesto, fundamentalmente, por centros tecnológicos, laboratorios de universidades, clústeres, asociaciones industriales, incubadoras, agencias de innovación, centros de innovación y comunidades de innovación.

El objetivo de esta iniciativa de la Comisión Europea es el desarrollo de una red europea de DIH que asegure una amplia cobertura geográfica en toda Europa.

Actualmente, la SGIPYME está trabajando en la definición de criterios para la selección de los mejores DIH nacionales que podrían formar parte de la red europea de DIH, y con ello resultar beneficiarios de las ayudas de la UE previstas en el Programa Europa Digital.

\section{5. Área de apoyo a la adopción de la 14.0 por la industria}

Esta área tiene como objetivo impulsar la creación, dentro de las empresas, de una

\footnotetext{
10 https://ec.europa.eu/digital-single-market/en/digital-innovation-hubs
}

metodología de adaptación permanente a la rápida y constante evolución de las tecnologías 4.0, así como favorecer la adopción inicial de los habilitadores digitales por parte de las industrias, a través de incentivos a la inversión en ellos.

Las iniciativas y programas correspondientes a esta área se engloban dentro de la conocida como «marca» ACTIVA, y son los que se indican en los apartados siguientes.

\subsection{Herramienta de Autodiagnóstico Digital Avanzada (HADA) ${ }^{11}$}

HADA es una aplicación online que, a través de un cuestionario de 68 preguntas, permite a las empresas conocer cuál es su grado de madurez digital y su estado en el proceso de adopción de soluciones de Industria 4.0, así como tener una referencia respecto a las empresas de su mismo sector, región o tamaño.

Esta herramienta constituye el primer eslabón dentro del proceso de apoyo a la digitalización de la industria diseñado por la SGIPYME y, aunque su principal público objetivo son las empresas industriales (CNAE del 10 al 32), su registro está abierto a todo tipo de empresas.

Para establecer el grado de madurez de las empresas registradas se han definido seis niveles de madurez que establecen el grado de implantación de la Industria 4.0 en las organizaciones y el punto en que se encuentran en el proceso de transformación digital.

HADA analiza dieciséis áreas distintas que se corresponden con cinco dimensiones organizacionales de las empresas:

https://www.industriaconectada40.gob.es/programas-apoyo/ Paginas/HADA.aspx 


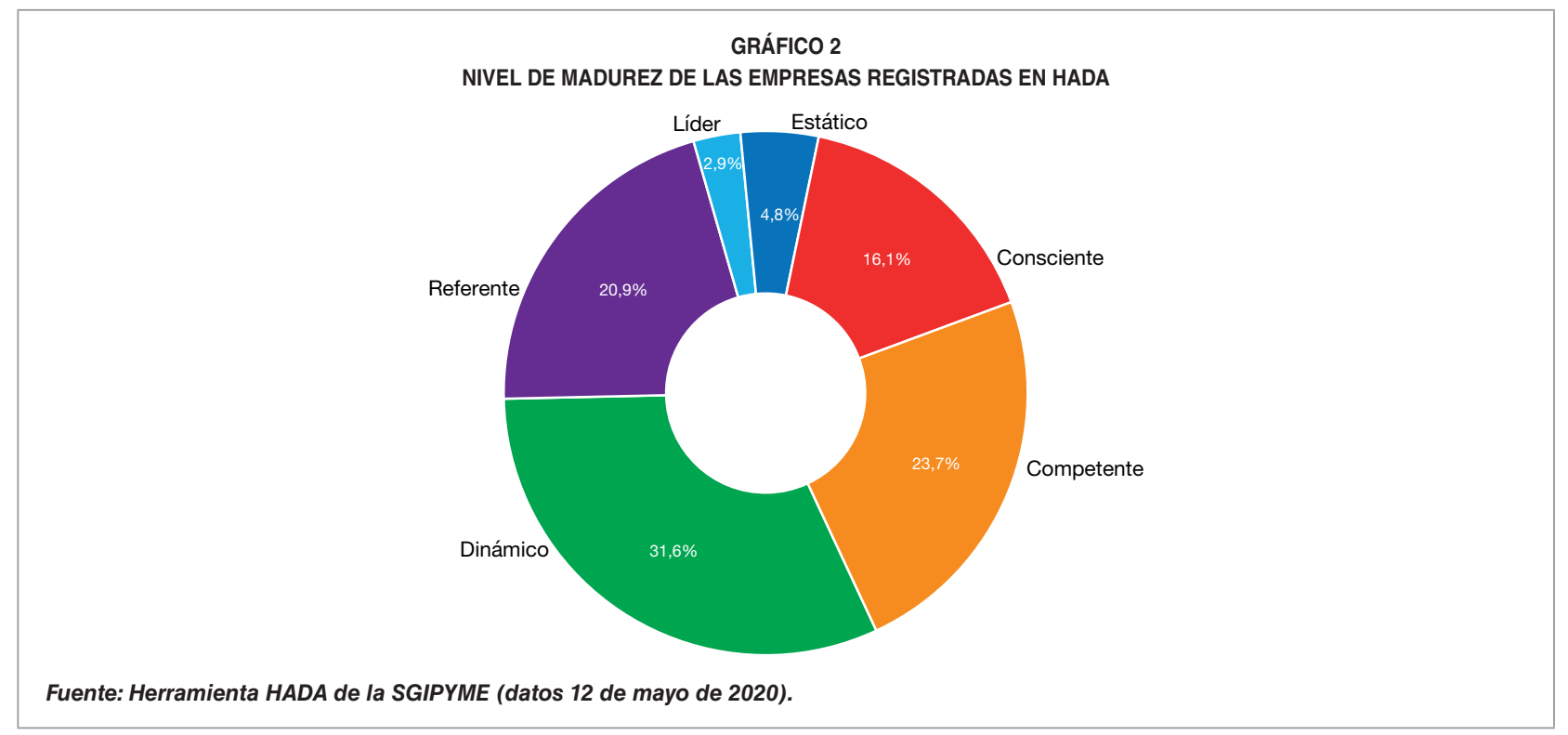

- Estrategia de mercado.

- Procesos.

- Organización y personas.

- Infraestructuras.

- Productos y servicios.

A fecha 12 de mayo de 2020, un total de 3.734 empresas están registradas en HADA, de las cuales un $68 \%$ ha completado la encuesta y, por lo tanto, ha obtenido su estado de madurez digital. De este cómputo total de empresas, 2.110 son empresas industriales (es decir, están englobadas en los CNAE 10 a 32), de las cuales un $74 \%$ ha finalizado la encuesta. El nivel de madurez de estas empresas está representado en el Gráfico 2.

\subsection{Programa ACTIVA Industria $4.0^{12}$}

ACTIVA Industria 4.0 es un programa de asesoramiento especializado y personalizado, realizado por consultoras acreditadas y con experiencia en implantación de proyectos de

12 https://www.industriaconectada40.gob.es/programas-apoyo/ Paginas/activa.aspx
Industria 4.0, y se realiza con la metodología desarrollada por la SGIPYME. Este programa permite a las empresas disponer de un diagnóstico de situación y de un plan de transformación que identifique los habilitadores digitales necesarios en ese proceso de transformación y establezca la hoja de ruta para su implantación. El asesoramiento se complementa con talleres demostrativos sobre tecnologías habilitadoras.

El programa es cofinanciado entre el Gobierno de España, las CC AA y las empresas beneficiarias. En su última edición, en el año 2019 , el programa contó con una dotación presupuestaria de 2,9 millones de euros para 280 asesoramientos, registrándose un total de 126 empresas participantes.

\subsection{Programa de Financiación Industria Conectada 4.0 (ACTIVA Financiación) ${ }^{13}$}

Esta actuación tiene como objetivo apoyar la incorporación de conocimientos, $\triangleright$

13 http://www.ipyme.org/es-ES/Financiacion/ApoyoFinan/nd/ Paginas/ApoyoFinanInd.aspx 
tecnologías e innovaciones destinadas a la digitalización de los procesos y a la creación de productos y servicios tecnológicamente avanzados y de mayor valor añadido en las empresas industriales.

El apoyo financiero tiene forma de préstamos reembolsables con las siguientes características:

- Importe máximo del préstamo: $80 \%$ del presupuesto financiable.

- Plazo máximo de amortización: diez años, incluyendo un periodo de carencia de tres años.

- Tipo de interés de aplicación: se establece en cada convocatoria, siendo igual o superior al tipo de interés euribor a un año, publicado por el Banco de España, correspondiente al mes anterior a la aprobación de la convocatoria o, en su caso, al mes anterior a su concesión.

- Presupuesto financiable mínimo de las actuaciones: 100.000 euros.

En la convocatoria del año 2018 contó con un presupuesto de 30 millones de euros y en 2019 de 50 millones de euros.

\subsection{Programa ACTIVA Ciberseguridad ${ }^{14}$}

Se trata de un programa cuyo objetivo es que las pymes, ante el incesante aumento de los ciberataques, determinen su nivel de seguridad actual y establezcan el nivel que han de conseguir para proteger sus sistemas y la información corporativos. El programa piloto fue lanzado en 2019 en colaboración con el Instituto

\footnotetext{
14 https://www.industriaconectada40.gob.es/programas-apoyo/ Paginas/ACTIVA-Ciberseguridad.aspx
}

Nacional de Ciberseguridad (INCIBE) y la EOI; en él han resultado beneficiarias 96 empresas.

El programa consta de cuatro fases complementarias dirigidas a pymes con domicilio social en cualquier provincia del territorio nacional:

- Fase 1 de autodiagnóstico inicial: recogida de información de la empresa y su sector y análisis de la situación actual de la empresa en materia de ciberseguridad para detectar las necesidades y posibilidades de mejora.

- Fase 2 de diagnóstico: análisis de cumplimiento / auditoría de ciberseguridad.

- Fase 3 de implantación: implantación de un plan de ciberseguridad en la empresa.

- Fase 4 de seguimiento: seguimiento de las medidas implantadas y valoración de otras iniciativas.

\subsection{Programa de Crecimiento Empresarial ${ }^{15}$}

Es un programa de consultoría personalizada consistente en 50 horas de asesoramiento que actúan sobre seis palancas de crecimiento: innovación, recursos humanos, operaciones, digitalización, marketing y comercialización y finanzas.

Cada proyecto de consultoría se divide en tres fases:

- Diagnóstico: análisis de la situación de partida para detectar posibilidades de mejora.

- Asesoramiento: selección de una de las palancas de crecimiento y propuesta $\triangleright$

15 https://www.industriaconectada40.gob.es/programas-apoyo/ Paginas/crecimiento-empresarial.aspx 
de acciones de mejora, con la elaboración del Plan de Crecimiento.

- Seguimiento: análisis periódico de indicadores de evaluación de resultados, con posterior incorporación al Club Crecimiento.

El programa va dirigido a pymes industriales con instalaciones productivas localizadas en España.

Desde su puesta en marcha en 2015, el programa ha ido creciendo. En la convocatoria de 2018 el programa contó con un presupuesto de 1,3 millones de euros y en 2019, de 1,7 millones de euros, siendo en total 229 y 285 empresas beneficiarias, respectivamente.

\section{6. Área de marco regulatorio y estandarización}

La incorporación de las tecnologías de la información a la industria ha supuesto que el sector industrial se enfrente a un nuevo escenario que plantea multitud de retos. Por ello, desde la Dirección General de Industria y de la Pyme del Ministerio de Industria, Comercio y Turismo, en el ámbito de la Estrategia Industria Conectada 4.0, se consideró conveniente el desarrollo de una serie de documentos normativos que sirvieran de base para la correcta transformación digital de nuestras empresas industriales.

En colaboración con UNE, diversas empresas y asociaciones empresariales, se han desarrollado dos especificaciones que definen los requisitos básicos a cumplir por parte de una empresa industrial para que pueda ser considerada como «industria digital».

Con estas especificaciones se persigue un doble objetivo: por un lado, que las empresas conozcan los requisitos que la economía y la sociedad demandan para ser consideradas empresas digitales; $y$, por otro, que puedan certificarlo de cara a clientes y proveedores, como sucede con las normas de calidad o de medio ambiente. Estas especificaciones se indican a continuación.

Especificación UNE 0060: 2018 Industria 4.0. Sistema de gestión para la digitalización. Requisitos

La Especificación UNE 0060, alineada con los ejes fundamentales de HADA, tiene por objeto describir los requisitos para que una empresa industrial de cualquier tamaño y/o actividad sea considerada como industria digital.

Este documento se articula a través de las distintas dimensiones empresariales (estrategia de negocio y mercado; productos y servicios; procesos; organización y personas e infraestructuras) y establece los indicadores que deben ser valorados adjuntando las explicaciones y definiciones oportunas para su correcto seguimiento e interpretación.

Estos requisitos, que posteriormente se clasificarán en obligatorios y valorables, comprenden todos los ámbitos organizacionales, desde las tecnologías habilitadoras hasta perfiles digitales, pasando por el establecimiento de una estrategia de digitalización a nivel empresarial, buscando que la transformación digital sea integral e impacte en la cultura organizacional y no solo en la elaboración de productos o servicios con el uso intensivo de tecnologías digitales.

Especificación UNE 0061:2019 Industria 4.0. Sistema de gestión para la digitalización. Criterios para la evaluación de requisitos

La Especificación UNE 0061 establece los criterios detallados para evaluar el $\triangleright$ 
cumplimiento de los requisitos definidos en la Especificación UNE 0060 y los criterios mínimos de cumplimiento de requisitos para conseguir la consideración de industria digital; es decir, establece el procedimiento de evaluación de conformidad con los requisitos previamente definidos.

Los requisitos se establecen como obligatorios y valorables. Los obligatorios son todos aquellos que se han considerado para establecer un nivel mínimo de madurez digital necesario en un escenario de Industria 4.0, mientras que los valorables suponen añadir experiencia y liderazgo en la transformación digital y se irán convirtiendo en obligatorios conforme avanzan los ciclos de mejora.

Esta norma define y establece un ciclo de mejora continua para que las empresas puedan ir implementando soluciones digitales a lo largo de distintos periodos en función de los requisitos.

Desde febrero de 2020 ambas especificaciones están a disposición de todas las empresas de manera gratuita a través de la página web de la Estrategia Nacional de Industria Conectada $4.0^{16}$.

\section{7. Área de Proyectos de $\mathbf{1 4 . 0}$}

Esta área tiene como objetivo desarrollar proyectos específicos de utilidad general, a través de mecanismos de colaboración público-privada, en código abierto para que se beneficien las empresas españolas, así como demostradores de ejemplos reales de aplicación de tecnologías habilitadoras.

\footnotetext{
16 https://www.industriaconectada40.gob.es/estrategias-informes/ Paginas/especificaciones-une.aspx
}

\subsection{Programa 12 Retos de la Industria $4.0^{17}$}

Este programa tiene el objetivo de conectar empresas de nueva generación, startups, con reconocidas empresas industriales en España y fomentar así la innovación abierta.

El proyecto está cofinanciado por el Ministerio de Industria, Comercio y Turismo, y por el Fondo Social Europeo.

Tras la identificación de los retos tecnológicos de doce grandes empresas industriales, la convocatoria busca seleccionar otras tantas startups que den respuesta a los mismos, mediante soluciones basadas en los habilitadores digitales de la Industria 4.0.

Para facilitar la obtención de esas soluciones, el programa ofrece a las startups seleccionadas un espacio virtual de aceleración con zonas comunes para reuniones, networking y lugares que facilitan el aprendizaje compartido. Además, dispondrán de mentoring y acompañamiento individualizado, así como de formación específica sobre aquellos aspectos que resulten cruciales para abordar los temas clave relacionados con el reto elegido.

La finalidad última del proyecto es impulsar modelos de innovación abierta y llegar a tener doce casos de éxito de colaboración entre empresa y startups que ayuden al crecimiento y consolidación de los emprendedores participantes en el programa.

La primera edición de este programa se celebró en 2019, y al final se resolvieron nueve de los doce retos lanzados, y con un total de ocho empresas que firmaron convenios de colaboración para implantar las soluciones obtenidas.

7 https://www.industriaconectada40.gob.es/programas-apoyo/ Paginas/12-retos-Industria.aspx 


\section{Conclusión}

La digitalización constituye una oportunidad clave para la mejora de la competitividad de la industria española en un mercado cada vez más global. Prácticamente todas las economías de nuestro entorno también han articulado estrategias nacionales para aprovecharla. Nuestra estrategia nacional aborda una profunda modificación para la industria española donde la transformación digital es clave para que las empresas españolas puedan abordar el reto y aprovechar las oportunidades que brinda la Industria 4.0, así como para evolucionar y posicionarse como un sector fuerte, competitivo y de referencia internacional.

\section{Bibliografía}

Carrasco Morales, M. (2019) Los congresos de industria conectada 4.0: génesis, evolución y futuras líneas de actuación. Revista de Economía Industrial (414), 143-145. https://www.mincotur. gob.es/Publicaciones/Publicacionesperiodicas/ Economialndustrial/RevistaEconomialndustrial/414/SEGUNDA\%20NOTA.pdf

Ministerio de Industria, Comercio y Turismo (2015). Estrategia Nacional Industria Conectada 4.0. https://www.industriaconectada40.gob.es/estrategias-informes/estrategia-nacional-IC40/Paginas/descripcion-estrategia-IC40.aspx

Ministerio de Industria, Comercio y Turismo. Página web Industria Conectada 4.0. España. https:// www.industriaconectada40.gob.es/Paginas/index.aspx 
